\title{
Prognostic Example of Stochastic Approach
}

\author{
Wolodymyr Hlib KOZYRSKI
}

\author{
The Bogolubov Institute for Theoretical Physics, \\ Ukrainian National Academy of Sciences, Kiev, 03680, Ukraine \\ *Corresponding Author: kozyrski@ukr.net
}

Copyright (C)2014 Horizon Research Publishing All rights reserved.

\begin{abstract}
The paper contains an application of Gonchar banking model to estimate default probability under random claim attack. We model investment environment by a sequence of random values characterizing risks under investing. Constructed model takes into account receipt of deposits to the accounts of bank and obligations fulfilment to return deposits with interest and other liabilities too. We model repayments by sequence of independent equally distributed random values depending on liabilities that can be fixed in various way. Constructed model is Markov homogeneous chain with transition function for one step that determined by probabilities of investment risk and distribution function of repayments. We consider a case when the distribution of payments has a "non-zero tails." We proved constructive theorems evaluating the probability of bankruptcy. The estimate depends on the probability of the capital loss due to systematic risk, the value of bad loans, initial capital, a minimum level of profitability, and determines the initial capital value for which the bank can operate without bankrupting for a long time under a relatively low probability of systematic risk. We have obtained the explicit expression for such initial capital. Therefore, Gonchar banking model lays a solid ground to estimate bank default risk in practice.
\end{abstract}

Keywords Banking, Bankruptcy, Financial Stability, N.S. Gonchar Banking Model, Random Claim Attack

\section{Introduction}

It is well known that many mathematical objects and techniques play surprisingly effective role describing both physical and non-physical phenomena. In particular, it concerns stochastic processes and fields being both a subject and a method to study some problems in statistical mechanics. As it turned out, they are also an effective mean to investigate topical problems in modern economy.

The economic critical events of the new century beginning and essentially their manifestations in the area of the global financial system is rather teasing topic both to today world and economic science.

Correct and authentic understanding of what is happening in the economy is a precondition for the proper problem formulation and searching reasonable precaution solution.

Problems of the financial bank stability possesses a special place in the field. In general, the problem of the bank stability under force majeure situations with abnormally large payment claims has known extra financial instruments of regulation, especially jury constraints of financial activities and the introduction of external administration.

Nevertheless, the ability to determine preventive policy to avoid the bankruptcy danger is up to now the fundamental problem. Two fundamental aspects of banking complicate this problem. The first characteristic embodied banks inherent randomness of the request for payment. The second arises from the lack of realistic banking models.

Many crucial factors within the problem and original stochastic nature of the bank economic counteragents activity forces to model bank operating under conditions of uncertainty in environment reactions. First attempts to understand the essence of the reality have their origin in $[1]$.

The Cramer-Lundberg [2, 3] and the Merton [4] models were the next step in clarifying the nature of risk operation results. VaR models and axiomatic risk estimations $[5,6]$ dominate in modern references.

Recently, we have seen a drastic progress in the second aspect. N. S. Gonchar suggested banking model [7] not only realistic in its features but also efficiently working and giving quantitative answers to the questions both about a precautionary bankruptcy assets and a possible term of bank stability under contingency requirements.

Here, we present the application of the dynamical model [7] of bank operating constructed by N.S.Gonchar as a practical consequence of his monograph [8] results.

Therefore, within the frame of N.S. Gonchar banking model [7], examples of realistic claims distribution function for bank payments are of particular interest in the context of bankruptcy, its warnings, and possible extension of the bank operating under demand attacks. Let us emphasize that it is a purely financial mechanisms without any external administrating. 


\section{The N.S. Gonchar Banking Model Formulation}

We will consider the following model of a bank operating.

Suppose that a bank invests in actives for one period a capital that it have at the beginning of the operation period. We assume that there are $n$ possible results of investment in every period of bank operating.

What possibility will realized depends on the state of investment environment and level of a bank management.

Suppose a bank operates in discrete time $t=1,2, \ldots$ in the following way: having an initial capital $x$ at time zero, within the next operating period bank invests in some assets so that can lose some capital with probability $p_{1}$ and can increase it with probability $p_{i}$, $i=\overline{2, N}$.

To describe this, introduce a sequence of independent identically distributed random variables $\varphi_{i}, \quad i=$ $1,2, \ldots, N$, having values in the set $\left\{b_{1}, \ldots, b_{N}\right\}$ and

$$
P\left(\varphi_{k}=b_{i}\right)=p_{i}>0, \quad \sum_{i=1}^{N} p_{i}=1
$$

where

$$
p_{k} \geq 0, \quad k=\overline{1, N}
$$

It is supposed that $0>b_{1}>-1, \quad b_{i}>b_{i-1} \geq 0$, $i=\overline{2, N}$.

Then bank capital at the time $t=1$ has the form

$$
R_{1}=x\left(1+\varphi_{1}\right)+Y_{1}-Z_{1}
$$

where $Y_{1}$ is nonnegative random variable describing amount of deposits entering the bank within first operating period and having values in the interval $\left[C_{0}, C\right]$, $C_{0} \geq 0,0<C<\infty, Z_{1}$ is nonnegative random quantity describing liability to creditors.

Operating in such a way, at the time $t=l$ bank shall have the capital

$$
R_{l}=R_{l-1}\left(1+\varphi_{l}\right)+Y_{l}-Z_{l}, \quad l=1,2, \ldots
$$

where the sequences of random variables $Y_{k}$ and $Z_{i}$, $k, i=1,2, \ldots$ are independent from each other and identically distributed. Also we suppose that sequences of random variables $\varphi_{i}, i=1,2, \ldots, Y_{k}, k=1,2, \ldots$, and $Z_{i}, i=1,2, \ldots$ are independent. The problem is to find bankruptcy probability of the bank under known $p_{i}, \quad i=\overline{1, N}$ and distribution function of random variables $Z_{n}$. One associates the constant $b_{1}$ with the problem bank credits.

\section{Default Probability Under Exponential Payments To The Bank Creditors}

Let consider the Banach space $\mathcal{B}_{\lambda}$ of Borel functions $f(x)$ with finite norm

$$
\|f\|_{\lambda}=\underset{x \in[0, \infty)}{\operatorname{ess} \sup } e^{\lambda x}|f(x)|, \quad \lambda>0
$$

In what follows $F(x)$ is a distribution function of bank liabilities payments.

For details see the paper [7].

Theorem 1. Let the conditions

$$
\begin{gathered}
1-F(x) \leq c_{1} e^{-a x}, \\
F(0)=0, c_{1}>0,
\end{gathered}
$$

hold where the constant $a>0$ is such that

$$
e^{-C \lambda}\left(1+c_{1} \lambda \int_{0}^{\infty} e^{-a y} e^{\lambda y} d y\right)<1
$$

Then the equation

$f(x)=\varphi(x)+\sum_{i=2}^{n} p_{i} \int_{0}^{\left(1+b_{i}\right) x+C} f\left[\left(1+b_{i}\right) x+C-y\right] d F(y)$

has unique solution in the space $\mathcal{B}_{\lambda}$ for arbitrary $\varphi(x) \in \mathcal{B}_{\lambda}$. For this solution, one has the estimate

$$
|f(x)| \leq \frac{e^{-\lambda x}\|\varphi\|_{\lambda}}{1-e^{-C \lambda}\left(1+c_{1} \lambda \int_{0}^{\infty} e^{-a x} e^{\lambda x} d x\right)}
$$

Proof. To prove the Theorem, let us estimate the linear operator

$$
A_{2} f=\sum_{i=2}^{n} \int_{0}^{\left(1+b_{i}\right) x+C} f\left[\left(1+b_{i}\right) x+C-y\right] d F(y)
$$

i.e.,

$$
\begin{gathered}
\left\|A_{2} f\right\|_{\lambda} \leq \\
\leq \underset{x \in[0, \infty)}{\operatorname{ess~sup}} e^{\lambda x} \sum_{i=2}^{n} p_{i} \int_{0}^{\left(1+b_{i}\right) x+C}\left|f\left[\left(1+b_{i}\right) x+C-y\right]\right| d F(y) \\
\leq \underset{x \in[0, \infty)}{\operatorname{ess~sup}} e^{\lambda x} \sum_{i=2}^{n} p_{i} \int_{0}^{\left(1+b_{i}\right) x+C} e^{-\lambda\left[\left(1+b_{i}\right) x+C-y\right]} d F(y)\|f\|_{\lambda} \\
\leq\left(1-p_{1}\right) \int_{0}^{\infty} e^{\lambda y} d F(y) e^{-\lambda C}\|f\|_{\lambda} .
\end{gathered}
$$


Due to the equality

$$
\int_{0}^{\infty} e^{\lambda y} d F(y)=1+\lambda \int_{0}^{\infty} e^{\lambda y}[1-F(y)] d y
$$

and the Theorem assumption, we obtain the estimate

$$
\int_{0}^{\infty} e^{\lambda y}[1-F(y)] d y \leq c_{1} \int_{0}^{\infty} e^{\lambda y} e^{-a y} d y .
$$

Therefore, $A_{2}$ is contraction operator with the norm not exceeding unit:

$$
\left(1-p_{1}\right) e^{-\lambda C}\left(1+\lambda c_{1} \int_{0}^{\infty} e^{\lambda y} e^{-a y} d y\right)<1
$$

The validity of the Theorem follows from the last estimate.

The next Theorem guarantees sufficient conditions to obtain bank default probability estimate.

\section{Theorem 2. Let}

$$
\lambda_{0}>0, \quad \lambda_{i}=\left(1+b_{1}\right)^{i} \lambda_{0}, \quad i=\overline{0, n},
$$

and for some $a>0$ there are inequalities

$$
\begin{gathered}
1+\lambda_{0} c_{1} \int_{0}^{\infty} e^{\lambda_{0} y} e^{-a y} d y<e^{\lambda C}, \\
1-F(x) \leq c_{1} e^{-a x} .
\end{gathered}
$$

Then the set of integral equations

$$
\begin{gathered}
\psi_{2}^{0}(x)=\varphi_{2}^{0}(x)+A_{2} \psi_{2}^{0}(x), \\
\psi_{2}^{0}(x)=A_{1} \psi_{2}^{i-1}(x)+A_{2} \psi_{2}^{i}(x), i=\overline{1, n} \\
\varphi_{2}^{0}(x)=\sum_{j=2}^{n} p_{j}\left\{1-F\left[\left(1+b_{j}\right) x+C\right]\right\} \\
A_{1} f(x)=p_{1} \int_{0}^{\left(1+b_{1}\right) x+C} f\left[\left(1+b_{1}\right) x+C-y\right] d F(y)
\end{gathered}
$$

has unique solution $\psi_{2}^{i}(x)$ satisfying inequalities

$$
\begin{gathered}
\psi_{2}^{i}(x) \leq D_{i} e^{-\lambda_{i} x}, \\
i=\overline{0, n},
\end{gathered}
$$

where

$$
\begin{gathered}
D_{i}= \\
=\frac{p_{1} e^{-\lambda_{i-1} C}\left(1+c_{1} \lambda_{i-1} \int_{0}^{\infty} e^{-a y} e^{\lambda_{i-1} y} d y\right)}{1-e^{-\lambda_{0}} C\left(1+c_{1} \lambda_{i} \int_{0}^{\infty} e^{-a y} e^{\lambda_{i} y} d y\right)} D_{i-1},
\end{gathered}
$$

$$
D_{0}=e^{-a C} .
$$

Proof. We prove the statement by induction in equations number. Supposing that for $i-1$ equations Theorem statement holds, consider the equation

$$
\psi_{2}^{i}(x)=A_{1} \psi_{2}^{i-1}(x)+A_{2} \psi_{2}^{i}(x) .
$$

By the statement of the Theorem 1, the last equation has unique solution in the Banach space $\mathcal{B}_{\lambda}$ if $A_{1} \psi_{2}^{i-1}(x)$ belongs to the space $\mathcal{B}_{\lambda_{i}}$. By the assumption,

$$
\psi_{2}^{i-1}(x) \leq D_{i-1} e^{-x \lambda_{i-1}} .
$$

From here, it follows that $A_{1} \psi_{2}^{i-1}(x)$ do belongs to the space $\mathcal{B}_{\lambda_{i}}$. Really,

$$
\begin{gathered}
A_{1} \psi_{2}^{i-1}(x) \leq \\
\leq p_{1} \int_{0}^{\left(1+b_{1}\right) x+C} e^{-\lambda_{i-1}\left[\left(1+b_{1}\right) x+C-y\right]} d F(y) D_{i-1} .
\end{gathered}
$$

In its turn,

$$
\begin{aligned}
& D_{i-1} e^{-\lambda_{i-1} C} p_{1} e^{-\lambda_{i-1}\left(1+b_{1}\right) x} \int_{0}^{\left(1+b_{1}\right) x+C} e^{\lambda_{i-1} y} d F(y) \\
& \leq D_{i-1} e^{-\lambda_{i-1}\left[C+\left(1+b_{1}\right) x\right]} p_{1}\left(1+c_{1} \int_{0}^{\infty} \lambda_{i-1} e^{\left(-a+\lambda_{i-1}\right) y} d y\right)
\end{aligned}
$$

By the Theorem 1, there exists unique solution in the space $\mathcal{B}_{\lambda_{i}}, \quad \lambda_{i}=\left(1+b_{1}\right) \lambda_{i-1}$, for which the inequality

$$
\psi_{2}^{i}(x) \leq
$$

$$
\leq \frac{e^{-\lambda_{i} x} p_{1} e^{-\lambda_{i-1} C}\left(1+c_{1} \lambda_{i-1} \int_{0}^{\infty} e^{-a y} e^{\lambda_{i-1} y} d y\right)}{1-e^{-\lambda_{i} C}\left(1+c_{1} \lambda_{i} \int_{0}^{\infty} e^{-a y} e^{\lambda_{i} y} d y\right)} D_{i-1}
$$

holds.

Introducing the abbreviation

$$
D_{i}=U_{i} D_{i-1}
$$

where

$$
U_{i}=\frac{p_{1} e^{-\lambda_{i-1} C}\left(1+c_{1} \lambda_{i-1} \int_{0}^{\infty} e^{-a y} e^{\lambda_{i-1} y} d y\right)}{1-e^{-\lambda_{i} C}\left(1+c_{1} \lambda_{i} \int_{0}^{\infty} e^{-a y} e^{\lambda_{i} y} d y\right)},
$$

we obtain the Theorem statement explicitly because the equation $(\star)$ by the Theorem 1 has unique solution in the space $\mathcal{B}_{\lambda_{i}}$ with the constant $D_{0}=e^{-a C}$.

Theorem 3. Let for some $a>0$ there are inequalities

$$
1-F(x) \leq c_{1} e^{-a y}
$$


and

$$
1+c_{1} \lambda_{0} \int_{0}^{\infty} e^{-a y} e^{\lambda_{0} y}<e^{-\lambda_{n} C} .
$$

Then bank default probability in l steps must satisfy the inequality

$$
\begin{gathered}
\psi_{l}(x) \leq p_{1}+\sum_{i=0}^{l} D_{i} e^{-\lambda_{i} x} \\
\lambda_{i}=\left(1+b_{1}\right)^{i} \lambda_{0} .
\end{gathered}
$$

There is such capital value $x_{0}$ that for arbitrary $\varepsilon>0$ the estimate

$$
\sum_{i=0}^{l} D_{i} e^{-\lambda_{i} x_{0}}<\varepsilon
$$

holds.

One proves the Theorem on the ground of the Lemma 3 and Theorem 7 by N.S. Gonchar in the paper [7].

Lemma (Lemma 3 from [7]). Suppose that $b_{1}>0$ and $P(\zeta=0)=0$.

For every $\omega \in\{\zeta \leq 0\}$ there exists a finite $n(\omega)<\infty$ such that

$$
\lim _{n \rightarrow \infty} \prod_{i=1}^{n} \chi_{[0, \infty)}\left(R_{i}\right)=\prod_{i=1}^{n(\omega)} \chi_{[0, \infty)}\left(R_{i}\right),
$$

and for every $\omega \in\{\zeta>0\}$

$$
\lim _{n \rightarrow \infty} \prod_{i=1}^{n} \chi_{[0, \infty)}\left(R_{i}\right)=0 .
$$

Proof. From the equality

$$
\chi_{[0, \infty)}\left(R_{k}\right)=\chi_{[0, \infty)}\left(x-\sum_{i=1}^{k} \frac{\eta_{i}}{\left(1+b_{1}\right)^{i}}\right)
$$

where $\eta_{i}$ denotes the quantity $Z_{i}-Y_{i}, i=\overline{0, \infty}$ and the fact that for every $\omega \in \Omega$

$$
\zeta=\lim _{n \rightarrow \infty} \frac{\sum_{i=1}^{n} \frac{\eta_{i}}{\left(1+b_{1}\right)^{i}}}{\sum_{i=1}^{n} \frac{1}{\left(1+b_{1}\right)^{i}}}
$$

exists we obtain that for all $\omega \in\{\zeta<0\}$ there exists $n(\omega)<\infty$ such that for all $n>n(\omega)$

$$
\frac{\sum_{i=1}^{n} \frac{\eta_{i}}{\left(1+b_{1}\right)^{i}}}{\sum_{i=1}^{n} \frac{1}{\left(1+b_{1}\right)^{i}}}>0 .
$$

This proves the first statement.

If $\omega \in\{\zeta>0\}$, then

$$
\lim _{n \rightarrow \infty} \sum_{i=1}^{n} \frac{\eta_{i}}{\left(1+b_{1}\right)^{i}}=-\infty .
$$

This proves the Lemma.
Theorem (Theorem 7 from [7]) Suppose that $1>p_{1}>0$.

For the probability of bankruptcy $\psi(x)$ in time interval $[0, \infty)$ with the initial capital $x$, the following representation and inequalities

$$
\begin{gathered}
\psi(x)=\sum_{n=0}^{\infty}\left[\psi_{1}^{(n)}(x)+\psi_{2}^{(n)}(x)\right], \\
\sum_{k=0}^{n-1} A^{k} \varphi_{0}(x) \leq \sum_{k=0}^{n}\left[\psi_{1}^{(k)}(x)+\psi_{2}^{(k)}(x)\right] \leq \psi(x), \\
n=1,2, \ldots,
\end{gathered}
$$

hold, where

$$
\begin{gathered}
\psi_{1}^{(0)}(x)=\varphi_{1}^{0}(x)+A_{1} \psi_{1}^{(0)}(x), \\
\psi_{2}^{(0)}(x)=\varphi_{2}^{0}(x)+A_{2} \psi_{2}^{(0)}(x), \\
\psi_{1}^{(n)}(x)=A_{2} \psi_{1}^{(n-1)}(x)+A_{1} \psi_{1}^{(n)}(x), \\
\psi_{2}^{(n)}(x)=A_{1} \psi_{2}^{(n-1)}(x)+A_{2} \psi_{2}^{(n)}(x), \\
\varphi_{1}^{0}(x)=p_{1}\left[1-F\left(\left(1+b_{1}\right) x+C\right)\right], \\
\varphi_{2}^{0}(x)=\sum_{j=2}^{n} p_{j}\left[1-F\left(\left(1+b_{j}\right) x+C\right)\right], \\
A_{2} f(x)=\sum_{j=2}^{n} p_{j}^{\left(1+b_{1}\right) x+C} \int_{0}^{\left(1+b_{j}\right) x+C} f\left(\left(1+b_{1}\right) x+C-y\right) d F(y), \\
\left.\int_{1} f(x)=p_{1} f\left(1+b_{j}\right) x+C-y\right) d F(y) .
\end{gathered}
$$

Proof. From the relation

$$
\begin{gathered}
\left(A_{1}+A_{2}\right)\left[\psi_{1}^{(n)}(x)+\psi_{2}^{(n)}(x)\right]= \\
=\psi_{1}^{(n)}(x)+\psi_{2}^{(n)}(x)-A_{2} \psi_{1}^{(n-1)}(x)+A_{2} \psi_{1}^{(n)}(x) \\
+A_{1} \psi_{2}^{(n)}(x)-A_{1} \psi_{2}^{(n-1)}(x)
\end{gathered}
$$

we obtain

$$
\begin{gathered}
\sum_{k=1}^{n}\left(A_{1}+A_{2}\right)\left[\psi_{1}^{(k)}(x)+\psi_{2}^{(k)}(x)\right]= \\
=\sum_{k=1}^{n}\left[\psi_{1}^{(k)}(x)+\psi_{2}^{(k)}(x)\right]-
\end{gathered}
$$


$-A_{2} \psi_{1}^{(0)}(x)-A_{1} \psi_{2}^{(0)}(x)+A_{2} \psi_{1}^{(n)}(x)+A_{1} \psi_{2}^{(n)}(x)$.

Therefore, we have

$$
\begin{aligned}
& \varphi_{1}^{(0)}(x)+\varphi_{2}^{(0)}(x)+\sum_{k=0}^{n}\left(A_{1}+A_{2}\right)\left[\psi_{1}^{(k)}(x)+\psi_{2}^{(k)}(x)\right] \\
& =\sum_{k=0}^{n}\left[\psi_{1}^{(k)}(x)+\psi_{2}^{(k)}(x)\right]+A_{2} \psi_{1}^{(n)}(x)+A_{1} \psi_{2}^{(n)}(x),
\end{aligned}
$$

or

$$
\begin{aligned}
& \varphi_{1}^{(0)}(x)+\varphi_{2}^{(0)}(x)+\sum_{k=0}^{n-1}\left(A_{1}+A_{2}\right)\left[\psi_{1}^{(k)}(x)+\psi_{2}^{(k)}(x)\right] \\
& =\sum_{k=0}^{n}\left[\psi_{1}^{(k)}(x)+\psi_{2}^{(k)}(x)\right]-A_{2} \psi_{2}^{(n)}(x)-A_{1} \psi_{1}^{(n)}(x) .
\end{aligned}
$$

From this expression we obtain the following inequalities

$$
\begin{gathered}
\varphi_{1}^{(0)}(x)+\varphi_{2}^{(0)}(x)+\sum_{k=1}^{n-1}\left(A_{1}+A_{2}\right)\left[\psi_{1}^{(k)}(x)+\psi_{2}^{(k)}(x)\right] \\
\geq \sum_{k=0}^{n}\left[\psi_{1}^{(k)}(x)+\psi_{2}^{(k)}(x)\right], \quad n=1,2, \ldots
\end{gathered}
$$

From the last inequalities we have the inequalities

$$
\sum_{k=0}^{n-1} A^{k} \varphi_{0}(x) \leq \sum_{k=0}^{n}\left[\psi_{1}^{(k)}(x)+\psi_{2}^{(k)}(x)\right], \quad n=1,2, \ldots
$$

By construction of $\psi_{i}^{(k)}(x)$, the inequalities

$$
\sum_{k=0}^{n}\left[\psi_{1}^{(k)}(x)+\psi_{2}^{(k)}(x)\right] \leq \psi(x), \quad n=0,1, \ldots
$$

hold.

From these inequalities and non-negativity of the probabilities $\psi_{1}^{(k)}(x)$ and $\psi_{2}^{(k)}(x)$, we obtain that there exists the limit

$$
\begin{gathered}
\lim _{n \rightarrow \infty} \sum_{k=0}^{n}\left[\psi_{1}^{(k)}(x)+\psi_{2}^{(k)}(x)\right] \\
=\sum_{k=0}^{\infty}\left[\psi_{1}^{(k)}(x)+\psi_{2}^{(k)}(x)\right]=\psi(x) .
\end{gathered}
$$

The last proves the theorem.

\section{Conclusions}

We have considered the situation when bank payments have "non-zero tails" and proved constructive Theorems that estimate default probability.
This estimation depends on capital loss probability through systematic risk, problem credits value, and starting capital.

We have obtained the explicit expression for the starting capital guaranteeing that the bank will operate without default for a long time under sufficiently small systematic risk probability.

As one can see, Gonchar bank operation model [7] lays a solid ground to estimate bank default risk in practice.

Therefore, it can be used not only in further academic investigations but also by bank analysts in their everyday practical activity.

\section{Acknowledgements}

Author thanks Prof. N. S. Gonchar for valuable discussions.

\section{REFERENCES}

[1] Altman E. Financial ratios, discriminant analysis and the prediction of corporate bankruptcy. // Journal of Finance. - vol.23. - P. 589-609. -1968.

[2] Cramer H. On the Mathematical Theory of Risk. // Skandia Jubilee Volume.-Stockholm. - 1930.

[3] Lundberg F. Some supplementary researches on the collective risk theory. //Skandinavisk Aktuarietidskrift. - vol.15. - P. 137-158. - 1932.

[4] Merton R. On the pricing of corporate debt: the risk structure of interest rates. // Journal of Finance. vol.29. - P. 449-470. - 1974.

[5] Kyshakevych B.Yu., Prykarpatsdky A.K., Tverdfokhlib I.P. Analysis of optimal strategies for a competing stock market portfolio model with a poilyvariant profit function // Cybernetics and Systems Analysis, Vol. 47, No. 2, March, 2011

[6] Artzner P., Delbaen F., Eber J.-M., Heath D. Coherent measures of risk. //Mathematical Finance. - vol.9, No.3. - P. 203-228. - 1999.

[7] Gonchar N. S. Bank capitalization Theorem // Journal of Automation Scinces, - V.44, - i2-60, - p. 65-80. 2012.

[8] Gonchar N.S. Mathematical Foundations of information economics. - K.: The Bogolubov Institute for Theoretical Physics, - 464 p. (in Ukrainian) - 2007.; 468 p. (in English) - 2008. 